\title{
ANÁLISE DE FATORES ASSOCIADOS À LESÃO DO NERVO LARÍNGEO RECORRENTE EM CIRURGIAS DE DISCECTOMIA CERVICAL VIA ANTERIOR
}

\author{
ANALYSIS OF FACTORS ASSOCIATED WITH LARYNGEAL NERVE INJURY IN ANTERIOR DISC \\ HERNIATION SURGERY \\ ANÁLISIS DE FACTORES ASOCIADOS A LA LESIÓN DEL NERVIO LARÍNGEO RECURRENTE \\ EN CIRUGÍAS DE DISCECTOMIIA CERVICAL POR VÍA ANTERIOR
}

Erasmo Abreu Zardo ${ }^{1}$, Afrane Serdeira ${ }^{2}$, Marcus Sofia Ziegler ${ }^{3}$, Joel Abramczuk ${ }^{4}$, Alexandre Coutinho Borba ${ }^{4}$

\begin{abstract}
RESUMO
Objetivo: Estudar os possíveis fatores associados com lesão do NLR após cirurgia de hérnia discal cervical com abordagem anterior. Métodos: No período de junho/2009 a junho/2010, avaliamos 30 pacientes submetidos a tratamento cirúrgico de hérnia discal via anterior no Hospital São Lucas da PUC-RS. No pré-operatório, foi realizada a medida da circunferência cervical (ao nível da cartilagem cricóide) e da altura cervical (do ângulo da mandíbula à borda superior da clavícula). No perioperatório, avaliamos o tempo e a dificuldade de entubação, o tempo cirúrgico, o lado da abordagem, o número de níveis operados, bem como o tipo de incisão (transversa/longitudinal) e o uso de halo craniano. Realizou-se uma avaliação videoendoscópica da laringe (VEL), em busca de lesão do NLR, no pré-operatório e no décimo dia após a cirurgia. Pacientes que apresentaram resultado anormal na VEL foram considerados com lesão do NLR e submetidos à reavaliação mensal até a recuperação espontânea ou no período máximo de seis meses quando a lesão foi considerada definitiva. Resultados: Encontramos 3/30 (10\%) casos de lesões não definitivas do NLR que se recuperaram em até 120 dias pós-operatórios. Os pacientes com lesão do NLR apresentaram uma maior circunferência do pescoço, tempo cirúrgico e número de níveis operados em relação aos pacientes sem lesão do NLR. Também, pacientes com lesão do NLR apresentaram um menor comprimento do pescoço. Duas lesões ocorreram na abordagem pelo lado direito e uma pelo lado esquerdo. Todos os pacientes com lesão tiveram incisão transversa e não fizeram uso de halo craniano. Conclusão: A abordagem pelo lado direito apresentou maior índice de complicações com o NLR. Apesar de o número limitado de pacientes não permitir conclusões estatisticamente significativas, fatores anatômicos intrínsecos do paciente como pescoço curto e diâmetro do pescoço aumentado, bem como tempo cirúrgico, e dificuldades técnicas que possam aumentar o tempo cirúrgico podem estar associados com lesão do NLR. Novos estudos avaliando as variáveis acima estudadas devem ser considerados.
\end{abstract}

Descritores: Abordagem cervical anterior/ lesão do nervo laríngeo recorrente.

\begin{abstract}
Objective: To study other possible factors associated with RLN injury after anterior approach for treatment of disc herniation. Methods: Between June 2009 and July 2010, we evaluated 30 patients underwent anterior approach for treatment of disc herniation in Hospital Sao Lucas, PUC-RS. At preoperative period, we evaluated the measure of neck circumference (at the level of the cricoid cartilage) and cervical height (angle of the jaw to the upper edge of the clavicle). At perioperative period, we evaluated the time and difficulty in endotracheal intubation, surgical time, the approach side, the number of levels operated, and the type of incision (transverse / longitudinal) and the use of the cranial halo. An endoscopic evaluation of the larynx was performed preoperatively. In the first 10 days after surgery, it was performed a second evaluation. The patients who had a normal second examination were considered without RLN injury. Patients who showed injuries were followed monthly until a period of six months when injuries were considered as definitive. Results: We found 3/30 (10\%) cases of temporary RLN injury which recovered to up to 120 days post operate. Related to patients with no RLN injury, these patients had a neck circumference greater than average, their surgical time was above average, as well the number of levels operated, and the neck length was shorter than average. Two injuries occurred in the approach through the right side and one in the left side. All patients with lesion had a transverse incision and no cranial halo was used. Conclusion: The approach from the right side showed a higher rate of complications in the RLN. A significant statistic conclusion was not possible due to the limited patients numbers; however, anatomical factors such as short neck and increased neck diameter, surgical time and other technical difficulties that can increase surgical time may be associated with RLN injury. New studies evaluating these factors must be considered.
\end{abstract}

Keywords: Anterior cervical approach/ recurrent laryngeal nerve injury.

RESUMEN

Objetivo: Estudiar los posibles factores asociados con la lesión del NLR postcirugía de hernia de disco cervical con abordaje anterior. métodos: En el periodo de Junio/2009 hasta Junio/2010 evaluamos 30 pacientes sometidos a tratamiento quirúrgico de hernia de disco por via anterior en el Hospital São Lucas de la PUC-RS. En el preoperatorio, fue realizada la medida de la circunferencia cervical (en el nivel

\footnotetext{
1 - Professor Doutor da Faculdade de Medicina da PUC-RS. Chefe do Serviço de Cirurgia da Coluna do Hospital São Lucas da PUC-RS - Rio Grande do Sul, Brasil.

2 - Ortopedista eTraumatologista Membro do Serviço de Cirurgia da Coluna do Hospital São Lucas da PUC-RS - Rio Grande do Sul, Brasil.

3 - Ortopedista eTraumatologista Membro do Serviço de Cirurgia da Coluna do Hospital São Lucas da PUC-RS - Rio Grande do Sul, Brasil.

4 - Ortopedista eTraumatologista Fellow do Serviço de Cirurgia da Coluna do Hospital São Lucas da PUC-RS - Rio Grande do Sul, Brasil.

Trabalho realizado no Serviço de Cirurgia da Coluna da Coluna do Hospital São Lucas da PUC - RS. Brasil.

Correspondência: Instituto Gaucho de Cirurgia da Coluna Vertebral. Av. Ipiranga,6690 - Sala 208 ,Centro Clinico da PUC-RS. CEP 90619-900. Partenon, Porto Alegre. RS. Brasil.

Email-contato@institutocoluna.com.br
} 
del cartílago cricoides) y de la altura cervical (del ángulo de la mandíbula borde superior de la clavicula). En el perioperatorio evaluamos el tiempo de intubación, el tiempo quirúrgico, el lado del abordaje, el número de niveles operados, bien como el tipo de incisión (transversal/ longitudinal) y el uso de halo craneano. Se realizó una evaluación videoendoscópica de laringe (VEL), en la búsqueda de lesión del NLR en el preoperatorio y en el décimo dia después de la cirugía. Pacientes que presentaron un resultado anormal de la VEL (asimetría de movimiento de las dobladuras al verbalizar las vocales A, E, I, O y U) fueron considerados con lesión del NLR y fueran reevaluadosmensualmente hasta la recuperación espontánea o en el periodo máximo de 6 meses, cuando la lesión fue considerada definitiva. Resultados: Encontramos $3 / 30(10 \%)$ casos de lesiones no definitivas del NLR que se recuperaron en hasta 120 dias postoperatorios. Los pacientes con lesión del NLR presentaban una mayor circunferencia del cuello, tiempo quirúrgico y número de niveles operados con relación a los pacientes sin lesión del NLR. También, pacientes con lesión del NLR presentaban una menor longitud del cuello. Dos lesiones ocurrieron en el abordaje por el lado derecho y una por el lado izquierdo. Todos los pacientes con lesión tuvieron incisión transversal y no hicieron (incisiones) de halo craneano.Conclusión: El abordaje por el lado derecho presentó mayor índice de complicaciones con el NLR. A pesar de que el número limitado de pacientes no permite conclusiones estadísticas significativas, factores anatómicos intrínsecos del paciente como cuello corto y diámetro aumentado, bien como tiempo quirúrgico y dificultades técnicas que puedan aumentarlo, pueden estar asociados a lesiones en el NLR. Nuevos estudios evaluando las variables arriba estudiadas deben ser considerados.

Descriptores: Abordaje cervical anterior/ lesión del nervio laríngeo recurrente.

\section{INTRODUÇÃO}

A abordagem cervical anterior para a coluna cervical é de uso freqüente pelos cirurgiões de coluna para tratamento de transtornos degenerativos, tumores e fraturas.

O lado escolhido para a abordagem bem como a relação do nível a ser abordado e a tração exercida pelos afastadores no transoperatório são apontados como as principais causas de lesão do NLR ( Lesão do Nervo Laríngeo).

Existem poucos trabalhos publicados correlacionando o risco de lesões com dificuldades de abordagem devido ao biótipo/anatomia do paciente ou mesmo do procedimento cirúrgico tais como: número de níveis operados e nível das lesões, tempo cirúrgico, tempo/dificuldade de entubação, tipo de incisão (transversa ou longitudinal) e uso de halo craniano. Nossa hipótese é que tais fatores podem estar relacionados com a ocorrência de lesão do NLR.

O objetivo deste estudo foi avaliar outros fatores, além dos já estabelecidos pela maioria de literatura médica, que podem contribuir para a lesão do NLR após cirurgia de hérnia discal cervical com abordagem anterior.

\section{MATERIAIS E MÉTODOS}

Entre Junho de 2009 à Julho de 2010, incluímos, de maneira consecutiva e não selecionada, 30 pacientes que foram submetidos a tratamento cirúrgico de hérnia discal via anterior no Hospital São Lucas da PUC-RS. A cirurgia realizada foi discectomia com colocação de Cage seguido ou não de placa. O plano de abordagem utilizado foi entre o músculo esternocleidomastóideo e a bainha carotídea lateralmente e os músculos da laringe e vísceras traqueoesofágicas medialmente. Os afastadores foram posicionados sob o músculo longus colli, após seu descolamento do corpo vertebral para evitar tração direta. A seleção para o lado da abordagem e uso de halo craniano e tipo de incisão foi aleatória.

O estudo foi aprovado pela comissão de ética do hospital e todos os pacientes assinaram um termo de consentimento livre e esclarecido. Todos os pacientes foram operados e anestesiados pela mesma equipe

\section{Os critérios de inclusão foram:}

- indivíduos com hérnia discal cervical posterior ou póstero lateral com indicação cirúrgica.

\section{Os critérios de exclusão foram:}

- indivíduos com cirurgias e/ou radioterapia prévias na região cervical ou outros traumatismos do pescoço;

- pacientes menores de 21 (vinte e um) anos de idade;

- pacientes que apresentavam lesão no NLR na primeira avaliação.
No pré-operatório, foi realizada a medida da circunferência cervical (ao nível da cartilagem cricóide) e da altura cervical (do ângulo da mandíbula à borda superior da clavícula). No perioperatório, avaliamos o tempo/dificuldade de entubação, o tempo cirúrgico, o lado da abordagem, o número de níveis operados, bem como o tipo de incisão (transversa/longitudinal) e o uso de halo craniano.

Uma avaliação videoendoscópia da laringe foi realizada no préoperatório. Nos primeiros 10 dias pós-operatórios, for realizada uma segunda avaliação. Os que tiveram um segundo exame normal foram considerados sem lesão do NLR. Aqueles que apresentaram lesão e recuperaram até seis meses foram considerados com lesão transitória. Após esse período, a lesão foi considerada definitiva.

\section{ANÁLISE ESTATÍSTICA}

As variáveis categóricas são apresentadas como percentagem e as variáveis numéricas pela sua média com desvio padrão. Para comparação entre frequências entre o grupo com ou sem lesão do NLR foram utilizados o teste de Pearson, e para comparação das médias entre os grupos, o teste $t$ student.

\section{RESULTADOS}

Entre os trinta pacientes incluídos no estudo 14(46,7\%) foram homens e 16(53,3\%) mulheres. A média idade foi de 46,0 8,7 anos e $3 / 30(10 \%)$ dos pacientes apresentaram lesão do NRL. Destes com lesão, dois $(66,6 \%)$ tiveram abordagem pelo lado direito e um $(33,3 \%)$ pelo lado esquerdo. Os dados foram concordantes com a literatura ${ }^{1-9}$. Não houve diferença significativa entre as variáveis estudadas no grupo com ou sem lesão (Tabela 1). No entanto, os pacientes com lesão tiveram uma circunferência do pescoço acima da média e um comprimento menor que a média em relação ao grupo sem lesão. Da mesma forma, o tempo cirúrgico, o tempo de entubação e o número de níveis operados foram acima da média. Nenhum paciente com lesão usou halo craniano e a incisão transversa foi usada em todos os casos.

\section{DISCUSSÂO}

A literatura estabelece que o menor comprimento do LNR direito o torna mais suscetível a lesões pelas forças de estiramento além do seu trajeto desprotegido pelo sulco traqueoesofágico ${ }^{10,11}$.

O mesmo tempo, se um paciente tem uma massa muscular maior e um pescoço mais curto as dificuldades cirúrgicas também aumentam, sendo necessário exercer uma força maior pelos afastadores para uma exposição adequada. Desse modo, o NLR também pode sofrer forças maiores de estiramento. Quando a cirurgia precisa ser 
Tabela 1. Associação entre as variáveis em estudo com a lesão.

\begin{tabular}{|c|c|c|c|}
\hline Variáveis & Com lesão (n=3) & Sem lesão (n=27) & $\mathbf{P}$ \\
\hline Idade (anos) - Média \pm DP & $52,3 \pm 2,1$ & $45,3 \pm 8,9$ & $0,185^{*}$ \\
\hline \multicolumn{4}{|l|}{ Sexo-n(\%) } \\
\hline Masculino & $0(0,0)$ & $14(51,9)$ & $0,228^{* *}$ \\
\hline Feminino & $3(100)$ & $13(48,1)$ & \\
\hline \multicolumn{4}{|l|}{ Níveis - n(\%) } \\
\hline 1 & $1(33,3)$ & $5(18,5)$ & $0,501^{* *}$ \\
\hline 2 & $3(100)$ & $20(74,1)$ & $1,000^{* *}$ \\
\hline 3 & $2(66,7)$ & $20(74,1)$ & $1,000^{* *}$ \\
\hline 4 & $0(0,0)$ & $1(3,7)$ & $1,000^{* *}$ \\
\hline \multicolumn{4}{|l|}{$\mathrm{N}^{0}$ de níveis $-\mathrm{n}(\%)$} \\
\hline 1 & $0(0,0)$ & $12(44,4)$ & $0,149 * * *$ \\
\hline 2 & $3(100)$ & $11(40,7)$ & \\
\hline 3 & $0(0,0)$ & $4(14,8)$ & \\
\hline \multicolumn{4}{|l|}{ Lado abordado - n(\%) } \\
\hline Direito & $2(66,7)$ & $11(40,7)$ & $0,565^{* *}$ \\
\hline Esquerdo & $1(33,3)$ & $16(59,3)$ & \\
\hline Tempo de cirurgia (min) - Média \pm DP & $125,3 \pm 7,2$ & $108,7 \pm 27,2$ & $0,307^{*}$ \\
\hline Circunferência cervical $(\mathrm{cm})-$ Média $\pm \mathrm{DP}$ & $37,7 \pm 0,6$ & $34,5 \pm 4,8$ & $0,684^{*}$ \\
\hline Altura cervical $(\mathrm{cm})-$ Média $\pm \mathrm{DP}$ & $13,0 \pm 4,4$ & $11,9 \pm 2,3$ & $0,482^{*}$ \\
\hline \multicolumn{4}{|l|}{ Incisão - n (\%) } \\
\hline Transversal & $3(100)$ & $14(51,9)$ & $0,238^{* *}$ \\
\hline Longitudinal & $0(0,0)$ & $13(48,1)$ & \\
\hline \multicolumn{4}{|l|}{ Halo craniano $-n(\%)$} \\
\hline Sim & $0(0,0)$ & $13(48,1)$ & $0,238^{* *}$ \\
\hline Não & $3(100)$ & $14(51,9)$ & \\
\hline Tempo de entubação (s) - Média \pm DP & $8,17 \pm 2,78$ & $8,13 \pm 3,33$ & $0,987^{*}$ \\
\hline
\end{tabular}

* Teste $t$-student para amostras independentes, ${ }^{* *}$ Teste exato de Fisher, *** Teste quiquadrado de Pearson.

realizada em mais de um nível, existe um maior movimento de reposicionamento dos afastadores que também podem contribuir para a lesão do LNR visto que ele não é de fácil visualização na abordagem anterior $^{12}$. O uso de halo craniano para obter maior distração trans operatória dos discos, indiretamente, aumenta a tensão da massa muscular cervical podendo aumentar o estiramento do NLR durante o afastamento. A incisão transversa é útil para a cirurgia realizada em poucos níveis. Para uma exposição mais ampla, deve ser utilizada a incisão longitudinal ${ }^{10}$. A escolha errada da incisão pode dificultar a exposição adequada facilitando a manipulação cega dos afastadores $^{12}$. A entubação traciona a laringe e, se houver alguma dificuldade maior, também pode agir como fator adjuvante para lesão do NLR.

\section{REFERÊNCIAS}

1. Beutler WJ, Sweeney CA, Connolly PJ. Recurrent laryngeal nerve injury with anterior cervical spine surgery risk with laterality of surgical approach. Spine (Phila Pa 1976). $2001 ; 26(12): 1337-42$

2. Apfelbaum RI, Kriskovich MD, Haller JR. On the incidence, cause, and prevention of recurrent laryngeal nerve palsies during anterior cervical spine surgery. Spine (Phila Pa 1976). 2000;25(22):2906-12.

3. Jung A, Schramm J, Lehnerdt K, Herberhold C. Recurrent laryngeal nerve palsy during anterior cervical spine surgery: a prospective study. J Neurosurg Spine.2005;2(2):123-7.

4. Tervonen H, Niemelä M, Lauri ER, Back L, Juvas A, Räsänen P, et al. Dysphonia and dysphagia after anterior cervical decompression. J Neurosurg Spine. 2007;7(2):124-30.

5. Yue WM, Brodner W, Highland TR. Persistent swallowing and voice problems after anterior cervical discectomy and fusion with allograft and plating: a 5- to 11-year follow-up study. Eur Spine J. 2005;14(7):677-82.

6. Fountas KN, Kapsalaki EZ, Nikolakakos LG, Smisson HF, Johnston KW, Grigorian AA, Lee GP, Robinson JS Jr. Anterior cervical discectomy and fusion associated complications. Spine (Phila Pa 1976). 2007;32(21):2310-7.

7. Kahraman S, Sirin S, Erdogan E, Atabey C, Daneyemez M, Gonul E. Is dysphonia permanent or temporary after anterior cervical approach? Eur Spine J. 2007;16(12):2092-5.

8. Kilburg C, Sullivan HG, Mathiason MA. Effect of approach side during anterior cervical
O maior tempo cirúrgico expõe o paciente a maior tempo de tração e aumenta o risco de lesão do NLR.

Existem poucos trabalhos na literatura que falam sobre fatores anatômicos intrínsecos do paciente, bem como dificuldades inerentes do procedimento cirúrgico como causa de lesão do NLR como foi considerado neste estudo. Cavo et al..$^{13} \mathrm{em}$ um trabalho de revisão relatou 30 casos de paralisia em pacientes que não realizaram cirurgia de cabeça, pescoço ou tórax todos atribuídos à entubação. Alguns estudos sugerem que a lesão por entubação ocorre pela pressão do cuff endotraqueal que pressiona o NLR contra a Lâmina tireoide ${ }^{14,15}$. Jellish et al. ${ }^{15}$, utilizando eletromiografia intra-operatória em 60 pacientes, encontrou mais alterações EMG em pacientes com o maior tempo de entubação, maior pressão do cuff e maior tempo cirúrgico. Apfelbaum et al. ${ }^{2}$, em um estudo com 900 casos, encontrou aumento progressivo do número de lesões de C5 a T1. Quanto ao número de níveis operados, encontrou diferença estatisticamente significativa quando comparada a cirurgia realizada em apenas um nível com a realizada em dois níveis ${ }^{2}$. Weisberg et a $/^{12}$, em um estudo em cadáveres, analisou o trajeto anatômico do NLR e levantou a hipótese de que incisões menores aumentam a possibilidade de retração cega dos tecidos podendo predispor a lesões do NLR.

Com base no aspecto anatômico e cirúrgico, nosso estudo avaliou fatores pouco considerados e os relacionou com a ocorrência de complicação do NLR após cirurgia de hérnia cervical. Apesar de aparentes diferenças nas variáveis estudadas entre pacientes com ou sem lesão, nossa pequena amostra não possibilitou que chegássemos a conclusões estatisticamente significativas. Mesmo assim, nossos dados apontam novos fatores possivelmente relacionados à complicação estudada e que devem ser considerados em novos estudos.

\section{CONCLUSÃO}

Encontramos maior prevalência de lesão do NLR na abordagem pelo lado direito.

Fatores anatômicos intrínsecos do paciente, como diâmetro e comprimento do pescoço e relacionados com a cirurgia como tempo cirúrgico e o número de níveis operados devem ser considerados em futuros estudos de lesão do NLR além dos habitualmente citados pela literatura.

\section{AGRADECIMENTOS}

Ao Professor Doutor Nédio Steffen do Serviço de Otorrinolaringologia do Hospital São Lucas da PUC-RS. discectomy and fusion on the incidence of recurrent laryngeal nerve injury. J Neurosurg Spine. 2006;4(4):273-7.

9. Netterville JL, Koriwchak MJ, Winkle M, Courey MS, Ossoff RH. Vocal fold paralysis following the anterior approach to the cervical spine. Ann Otol Rhinol Laryngol. 1996:105(2):85-91.

10. Chang U, Lee MC, Kim DH. Abordagem anterior para a coluna cervical media. In: Kim DH, Hem JS, Vaccaro AR, Dickman CA. Coluna vertebral anatomia e tecnicas cirúrgicas. Rio de Janeiro: Di Livros; 2007. p.50-1.

11. Moore KL. Pescoço. In: Moore KL, Dalley AF. Anatomia orientada para a clinica. Rio de Janeiro: Guanabarra Koogan; 2007. p. 1014-5.

12. Weisberg NK, Spengler DM, Netterville JL. Stretch-induced nerve injury as a cause of paralysis secondary to the anterior cervical approach. Otolaryngol Head Neck Surg. 1997:116(3):317-26.

13. Cavo JW Jr. True vocal cord paralysis following intubation. Laryngoscope.1985;95(11):1352-9.

14. Bulger RF, Rejowski JE, Beatty RA. Vocal cord paralysis associated with anterior cervical fusion: considerations for prevention and treatment. J Neurosurg. 1985;62(5):657-61.

15. Jellish WS, Jensen RL, Anderson DE, Shea JF. Intraoperative electromyographic assessment of recurrent laryngeal nerve stress and pharyngeal injury during anterior cervical spine surgery with Caspar instrumentation. J Neurosurg. 1999;91(2 Suppl):170-4 